Theoretical and Mathematical Physics, 208(3): 1317 (2021)

\title{
ERRATA
}

\section{Erratum to: EXPONENTIALLY CONFINING POTENTIAL WELL}

\author{
A. D. Alhaidari ${ }^{*}$
}

DOI: $10.1134 / S 0040577921090117$

Formula (16) should be

$$
\psi(x)=\sqrt{\rho\left(\frac{4 \varepsilon}{A_{+}}\right)} \sum_{n=0}^{N-1} B_{n}^{\mu}\left(\frac{4 \varepsilon}{A_{+}} ;-\frac{4}{A_{+}}\right) \phi_{n}(x),
$$

The editorial staff apologizes to the author.

The original article can be found online at https://doi.org/10.1134/S0040577921010050.

*Saudi Center for Theoretical Physics, Jeddah, Saudi Arabia, e-mail: a.d.alhaidari@gmail.com.

Received June 23, 2021. Revised June 23, 2021. Accepted June 23, 2021. 